TRANSACTIONS OF THE

AMERICAN MATHEMATICAL SOCIETY

Volume 354, Number 9, Pages 3703-3722

S 0002-9947(02)03035-0

Article electronically published on May 7, 2002

\title{
COMPACTNESS PROPERTIES \\ FOR FAMILIES OF QUASISTATIONARY SOLUTIONS OF SOME EVOLUTION EQUATIONS
}

\author{
GIUSEPPE SAVARÉ
}

\begin{abstract}
The following typical problem occurs in passing to the limit in some phase field models: for two sequences of space-time dependent functions $\left\{\theta_{n}\right\},\left\{\chi_{n}\right\}$ (representing, e.g., suitable approximations of the temperature and the phase variable) we know that the sum $\theta_{n}+\chi_{n}$ converges in some $L^{p}$-space as $n \uparrow+\infty$ and that the time integrals of a suitable "space" functional evaluated on $\theta_{n}, \chi_{n}$ are uniformly bounded with respect to $n$. Can we deduce that $\theta_{n}$ and $\chi_{n}$ converge separately? LuCKHAus (1990) gave a positive answer to this question in the framework of the two-phase Stefan problem with Gibbs-Thompson law for the melting temperature. Plotnikov (1993) proposed an abstract result employing the original idea of Luckhaus and arguments of compactness and reflexivity type. We present a general setting for this and other related problems, providing necessary and sufficient conditions for their solvability: these conditions rely on general topological and coercivity properties of the functionals and the norms involved, and do not require reflexivity.
\end{abstract}

\section{INTRODUCTION}

In this paper we are mainly interested in the following type of problem, involving vector-valued functions defined on the time interval $] 0, T[, T>0$ :

Problem $(\mathbf{P})$. Let us assume that $A, B$ are two Banach spaces,

$$
L: A \rightarrow B \text { is a bounded linear operator, }
$$

$$
\mathscr{F}: A \rightarrow[0,+\infty] \text { is a proper lower semi-continuous functional. }
$$

We are given a sequence $\left.u_{n}:\right] 0, T[\rightarrow A$ of time-dependent (strongly) measurable functions, such that

$$
\exists \lim _{n \uparrow+\infty} L u_{n}=: \ell \quad \text { in } L^{p}(0, T ; B) \quad \text { for some } p \in[1,+\infty] \text {; }
$$

Received by the editors October 3, 2001

2000 Mathematics Subject Classification. Primary 47J25, 80A22; Secondary 37L05, 47J35, 49J45.

Key words and phrases. Quasistationary solutions of evolution equations, phase-field models, Stefan problem with Gibbs-Thomson law, compactness methods.

This work was partially supported by the M.U.R.S.T. (Italy) through national research project funds, and by the Institute of Numerical Analysis of the C.N.R., Pavia, Italy.

(C)2002 American Mathematical Society 
moreover, we know an additional a priori estimate of the type

$$
S:=\sup _{n \in \mathbb{N}} \int_{0}^{T} \mathscr{F}\left(u_{n}(t)\right) d t<+\infty .
$$

Could we find $q \in[1,+\infty)$ and a suitable subsequence of $u_{n}$ strongly converging in $L^{q}(0, T ; A)$ ?

Of course, we are looking for some kind of general assumptions on $L, \mathscr{F}$ and, possibly, on the asymptotic behavior of $\left\{u_{n}\right\}_{n \in \mathbb{N}}$, which are sufficient to force the $L^{q}(0, T ; A)$-convergence.

Before entering into the details of our analysis, let us mention some basic examples which better explain and motivate the question we are considering.

Example 1. We start from an important problem solved by S. LuCKHAus [Luc90. Lemma 1,2] (see also Vis96. VIII.3]) in the framework of the two-phase StefanGibbs-Thomson model for the melting temperature. In the cylindrical region

$$
Q:=] 0, T\left[\times \Omega, \quad \Omega \text { being a bounded open subset of } \mathbb{R}^{3},\right.
$$

we are considering two sequences of real functions (they are suitable approximations of the temperature and the phase variable) $\theta_{n}, \chi_{n} \in L^{1}(Q)$ that satisfy

$$
\begin{gathered}
\left.\left|\chi_{n}(x, t)\right|=1 \quad \text { a.e. in } Q, \quad \theta_{n}(\cdot, t) \in H_{0}^{1}(\Omega) \quad \text { for a.e. } t \in\right] 0, T[, \\
\int_{Q}\left|\nabla_{x} \theta_{n}(x, t)\right|^{2} d x d t \leq S<+\infty,
\end{gathered}
$$

and

$$
\theta_{n}+\chi_{n} \rightarrow \ell \quad \text { strongly in } L^{3}\left(0, T ; L^{1}(\Omega)\right) \text { as } n \uparrow+\infty \text {. }
$$

Luckhaus succeeded in proving that (1.2), (1.3), (1.4) imply the separated strong convergence in $L^{1}(Q)$ of $\theta_{n}$ and $\chi_{n}$ to $\theta$ and $\chi$ respectively; this result was a cornerstone of the existence proof of solutions of the two-phase Stefan problem.

Let us show that this situation can be rephrased with the notation of Problem (P). We choose

$$
A:=L^{1}(\Omega) \times L^{1}(\Omega), \quad B:=L^{1}(\Omega), \quad p=3, q=1,
$$

and we consider the couple $u_{n}:=\left(\theta_{n}, \chi_{n}\right)$ as a time-dependent function with values in $A$; so we set

$$
L: u=(\theta, \chi) \in L^{1}(\Omega) \times L^{1}(\Omega) \rightarrow \theta+\chi \in L^{1}(\Omega),
$$

and we introduce the functionals, defined on $L^{1}(\Omega)$,

$$
\begin{gathered}
\mathscr{F}_{1}(\theta):= \begin{cases}\int_{\Omega}|\nabla \theta(x)|^{2} d x & \text { if } \theta \in V:=H_{0}^{1}(\Omega), \\
+\infty & \text { otherwise; }\end{cases} \\
\mathscr{F}_{2}(\chi):= \begin{cases}0 & \text { if }|\chi(x)|=1 \text { a.e. in } \Omega, \\
+\infty & \text { otherwise. }\end{cases}
\end{gathered}
$$

Finally we define

$$
\mathscr{F}(u):=\mathscr{F}_{1}(\theta)+\mathscr{F}_{2}(\chi) .
$$

The Luckhaus Theorem is then equivalent to saying that in the context of $(1.5), \ldots$, (1.9), Problem (P) has an affirmative solution. Even if the original proof of this 
statement relies on careful capacity-type estimates for Sobolev functions defined on $\Omega$, it is natural to look for a more abstract principle behind this result.

Example 2. A first step in this direction was provided by P.I. PlotniKov (see PS93, and also HS98a, HS98b for other applications), who considered the case of two time-dependent sequences

$$
n \mapsto \theta_{n}, \chi_{n} \in L^{p}(0, T ; B), \quad B \text { being a Banach space, }
$$

such that

$$
\theta_{n}+\chi_{n} \rightarrow \ell \quad \text { strongly in } L^{p}(0, T ; B) \text { as } n \uparrow+\infty .
$$

Moreover, he supposed that

$$
\begin{gathered}
\left.\theta_{n}(t) \in V, \quad \chi_{n}(t) \in K \quad \text { for a.e. } t \in\right] 0, T[, \\
\int_{0}^{T}\left\|\theta_{n}(t)\right\|_{V}^{p} d t \leq S<+\infty \quad \forall n \in \mathbb{N},
\end{gathered}
$$

where

$$
V \hookrightarrow B \text { is another Banach space, } K \text { is a closed subset of } B,
$$

satisfying the compatibility condition

$$
\chi, \chi^{\prime} \in K, \quad \chi-\chi^{\prime} \in V \quad \Rightarrow \quad \chi=\chi^{\prime} .
$$

It is easy to identify the choices of $p, B, V, K$ in (1.10), $, \ldots,(1.15)$ which correspond to (1.2), ..., (1.4); [PS93] proved that if

(1.16) $p=2, V, B$ are reflexive, $K$ is compact, the inclusion $V \subset B$ is compact,

then (1.10) $, \ldots,(1.15)$ entail the separated convergence of $\theta_{n}$ and $\chi_{n}$ in $L^{2}(0, T ; B)$ as $n \uparrow+\infty$.

As before, setting $A:=B \times B, L: u=(\theta, \chi) \rightarrow \theta+\chi, \mathscr{F}(u)=\mathscr{F}_{1}(\theta)+\mathscr{F}_{2}(\chi)$ with

$$
\mathscr{F}_{1}(\theta):=\left\{\begin{array}{ll}
\|\theta\|_{V}^{p} & \text { if } \theta \in V, \\
+\infty & \text { otherwise, }
\end{array} \quad \mathscr{F}_{2}(\chi):= \begin{cases}0 & \text { if } \chi \in K, \\
+\infty & \text { otherwise }\end{cases}\right.
$$

we are in the framework of Problem $(\mathrm{P})$; the lower semicontinuity of $\mathscr{F}$ is ensured by the closeness of $K$ and the reflexivity of $V$, which in particular entails its relative completeness in $B$

$$
\left.\begin{array}{c}
v_{n} \in V, \quad \sup _{n}\left\|v_{n}\right\|_{V}<+\infty \\
v_{n} \rightarrow v \text { strongly in } B
\end{array}\right\} \Rightarrow v \in V, \quad\|v\|_{V} \leq \liminf _{n \uparrow+\infty}\left\|v_{n}\right\|_{V} .
$$

The latter property, which is equivalent to saying that $V$ coincides with its Gagliardo completion in $B$ Gag61], is in fact weaker than reflexivity or compactness, which are the crucial assumptions (1.16) of [PS93]; moreover, these assumptions are not necessary in order to give a positive answer to Problem (P), as the Luckhaus Theorem shows. Our aim is to fill in the gap between these two results in the abstract context detailed before. 
Example 3. With the notation of Problem (P), let us assume in particular that $A$ is continuously embedded in $B, L$ is the corresponding inclusion map, and $p=q$. We consider another Banach space $V \subset A$ and we define $\mathscr{F}$ as

$$
\mathscr{F}(v):= \begin{cases}\|v\|_{V}^{p} & \text { if } v \in V \\ +\infty & \text { if } v \in A \backslash V\end{cases}
$$

assuming that it is lower semicontinuous. Therefore, for a given sequence $\left\{u_{n}\right\}_{n \in \mathbb{N}}$ which is uniformly bounded in $L^{p}(0, T ; V)$ as in $(\mathbb{P} .4)$, and converges in $L^{p}(0, T ; B)$ as in (P.3), we are asking if $u_{n}$ converges in $L^{p}(0, T ; A)$, too. It is not difficult to show that in this case the good condition is

$$
\forall \varepsilon>0, \exists C_{\varepsilon}>0: \quad\|v\|_{A} \leq \varepsilon\|v\|_{V}+C_{\varepsilon}\|v\|_{B} \quad \forall v \in V .
$$

Typically, two standard assumptions imply (1.19):

- $V$ is compactly embedded in $A$ [LM72, Ch. I, Th. 16.4],

- $A$ is an interpolation space between $V$ and $B$ of class $\underline{\mathcal{K}}_{\theta}(V, B)$, for $\theta \in(0,1]$ [LP64, Chap. IV, Def. 1.1], i.e., there exists a constant $C>0$ such that

$$
\|v\|_{A} \leq C\|v\|_{V}^{1-\theta}\|v\|_{B}^{\theta} \quad \forall v \in V,
$$

or, equivalently, the real interpolation space $(V, B)_{\theta, 1}$ is contained in $A$.

It is interesting to observe that (1.19) is in fact equivalent to saying that the topologies induced by the norms of $A$ and $B$ coincide on $V$-bounded sets, i.e.,

$$
\left.\begin{array}{l}
v_{k} \in V, \quad\left\|v_{k}\right\|_{V} \leq S<+\infty \\
v_{k} \rightarrow v \text { strongly in } B
\end{array}\right\} \Rightarrow v_{k} \rightarrow v \text { strongly in } A .
$$

Plan of the paper. In the next section we will present a preliminary discussion about the reasonable assumptions which seem to be necessary to solve Problem (P); afterward, in the same section, we will see that these assumptions are also sufficient, and we will collect the main abstract results, whose proofs are detailed in $\S 3$. Particular attention is devoted to the case of a "quasistationary" family $u_{n}$ satisfying an a.e. minimality condition with respect to another sequence of timedependent functionals.

Applications are presented in the last section: they concern an improved version of the Luckhaus and Plotnikov theorems and a convergence result for quasistationary solutions of phase field equations with Neumann boundary conditions: this last application refines, with different techniques, a recent result of R. SCHÄTzLE Sch00.

\section{Basic Assumptions and Main Results}

Preliminary discussion. Before stating the main theorems of this paper, we try to understand what kind of (hopefully minimal) assumptions could provide a reasonable answer to Problem $(\mathrm{P})$.

Compactness. Let us start with a sequence of constant functions

$$
\left.u_{n}(t):=v_{n} \in A \quad \text { for } t \in\right] 0, T[;
$$

in this case ( $(\mathrm{P} .3)$ and $(\mathrm{P} .4)$ can be reformulated in terms of the constants $v_{n}$ as

$$
\exists \ell \in B: \lim _{n \uparrow+\infty} L v_{n}=\ell \text { in } B, \quad \exists s<+\infty: \mathscr{F}\left(v_{n}\right) \leq s \quad \forall n \in \mathbb{N} .
$$


Since we are looking for a subsequence of $v_{n}$ converging in $A$ and we do not know other information on the sequence $v_{n}$ and the values of $\ell, s$, we are forced to impose a sort of conditional compactness on all the sublevels of $\mathscr{F}$. We adopt the following definition.

Definition 2.1. We say that a subset $K \subset A$ is conditionally compact w.r.t. $L$ if every sequence $n \mapsto v_{n} \in K$ such that $\exists \lim _{n \uparrow+\infty} L v_{n}$ in $B$ has a (strongly) convergent subsequence.

So we are led to assume that

$$
\begin{aligned}
& \text { The sublevels }\{v \in A: \mathscr{F}(v) \leq s\} \\
& \text { are conditionally compact w.r.t. } L, \quad \forall s \geq 0 \text {. }
\end{aligned}
$$

Let us observe that the compactness of the sublevels trivially implies (H.1).

Coercivity. The second assumption regards the asymptotic behavior of $\mathscr{F}(v)$ as $\|v\|_{A}$ goes to $+\infty$. Consider, e.g., the case $q=1$ : we want to show that, roughly speaking, we need a superlinear growth of $\mathscr{F}$, at least in the directions not controlled by $L$.

In order to clarify this point, let us suppose that the superlinear growth fails along a diverging sequence $\left\{v_{n}\right\}_{n \in \mathbb{N}}$ in $A$, whose asymptotic direction belongs to Ker $L$, i.e.,

$$
\lim _{n \uparrow+\infty}\left\|v_{n}\right\|_{A}=+\infty, \quad \sup _{n \in \mathbb{N}} \frac{\mathscr{F}\left(v_{n}\right)}{\left\|v_{n}\right\|_{A}}<+\infty, \quad \lim _{n \uparrow+\infty} L\left(\frac{v_{n}}{\left\|v_{n}\right\|_{A}}\right)=0 .
$$

Then we set

$$
t_{n}:=\left\|v_{n}\right\|_{A}^{-1}, \quad u_{n}(t):= \begin{cases}v_{n} & \text { if } 0<t<t_{n}, \\ v_{0} & \text { otherwise }\end{cases}
$$

and it is easy to see that, as $n \uparrow+\infty$,

$$
\begin{gathered}
\int_{0}^{T}\left\|L u_{n}(t)-L v_{0}\right\| d t \leq t_{n}\left(\left\|L v_{n}\right\|_{B}+\left\|L v_{0}\right\|_{B}\right) \rightarrow 0, \\
\sup _{n \in \mathbb{N}} \int_{0}^{T} \mathscr{F}\left(u_{n}(t)\right) d t \leq \sup _{n \in \mathbb{N}}\left(T \mathscr{F}\left(v_{0}\right)+t_{n} \mathscr{F}\left(v_{n}\right)\right)<+\infty,
\end{gathered}
$$

whereas

$$
\lim _{n \uparrow+\infty} \int_{0}^{T}\left\|u_{n}(t)-v_{0}\right\|_{A} d t=1 .
$$

As before, we introduce the crucial concept in the next definition, where we assume $q, p \in[1,+\infty)$.

Definition 2.2. We say that $\mathscr{F}$ is $(q, p)$-conditionally coercive in $A$ w.r.t. $L$ if

$$
\forall \varepsilon>0 \exists C_{\varepsilon}>0: \quad\|v\|_{A}^{q} \leq \varepsilon \mathscr{F}(v)+C_{\varepsilon}\left(1+\|L v\|_{B}^{p}\right) \quad \forall v \in A .
$$

Let us remark that, for $q=p=1$, (H.2) is exactly equivalent to assuming that sequences like (2.1) do not exist.

Nondegeneracy. The last kind of questions regards the injectivity of $L$ on the proper domain $D(\mathscr{F})$ of $\mathscr{F}$,

$$
D(\mathscr{F}):=\{v \in A: \mathscr{F}(v)<+\infty\} .
$$


If it happens that

$$
\exists v, w \in D(\mathscr{F}): \quad v \neq w, \quad L v=L w,
$$

then it is not difficult to exhibit a sequence of functions $u_{n}$ whose range spans $\{v, w\}$, which do not have any convergent subsequence. Indeed, we consider an orthogonal system $\left\{e_{n}\right\}_{n \in \mathbb{N}}$ in $L^{2}(0, T)$, constituted by functions assuming only the values $-1,1$ (e.g., the standard Haar system), and we set

$$
u_{n}(t):=\frac{1}{2}(v+w)+\frac{1}{2}(v-w) e_{n}(t) .
$$

Since

$$
L u_{n}(t) \equiv L v=L w \quad \text { is independent of } n,
$$

and

$$
\int_{0}^{T} \mathscr{F}\left(u_{n}(t)\right) d t \leq T \max \{\mathscr{F}(v), \mathscr{F}(w)\},
$$

it is easy to see that $(\underline{\mathrm{P} .3})$ and $(\mathrm{P} .4)$ are satisfied, with $p=+\infty$. On the other hand, a subsequence $k \mapsto u_{n_{k}}$ converging in $L^{1}(0, T ; A)$ would converge in every $L^{q}(0, T ; A), q<+\infty$; in particular, we should have

$$
k \mapsto u_{n_{k}} \text { is a Cauchy sequence in } L^{2}(0, T ; A),
$$

but the orthogonality of the system $\left\{e_{n}\right\}_{n \in \mathbb{N}}$ yields for $n, m \in \mathbb{N}, n \neq m$,

$$
\left\|u_{n}-u_{m}\right\|_{L^{2}(0, T ; A)}^{2}=\frac{1}{4}\|v-w\|_{A}^{2} \int_{0}^{T}\left|e_{n}(t)-e_{m}(t)\right|^{2} d t=\frac{T}{2}\|v-w\|_{A}^{2}>0 .
$$

We formalize the property we will need in the next definition.

Definition 2.3. We say that a subset $K \subset A$ is nondegenerate w.r.t. $L$ if

$$
v, w \in K, \quad L v=L w \quad \Rightarrow \quad v=w .
$$

Correspondingly, we will assume that

$$
D(\mathscr{F}) \text { is nondegenerate w.r.t. L. }
$$

Main results. The previous arguments show that properties (H.1), (H.2), and (․․3) are necessary in order to give a positive answer to Problem (P). The next theorem says that they are also sufficient; moreover, the convergence of the whole sequence $u_{n}$ is provided.

Theorem 1. Let us suppose that L, F satisfy (P.1), (P.2), and

(H.1) the sublevels of $\mathscr{F}$ are conditionally compact w.r.t. L,

(H.2) $\mathscr{F}$ is $(q, p)$-conditionally coercive in A w.r.t. L,

(H.3) $D(\mathscr{F})$ is nondegenerate w.r.t. L.

Then Problem $(\mathrm{P})$ has an affirmative answer, i.e., every sequence of functions $u_{n} \in L^{q}(0, T ; A)$ satisfying $(\mathbb{P . 3})$ and $(\mathbb{P . 4})$ is strongly convergent in $L^{q}(0, T ; A)$.

We postpone the proof of Theorem 1 to the next section; now we want to refine this result, in order to have a better insight in the case when (H.3) does not hold.

The possibly degenerate case. In some situation (H.3) could be false, but nevertheless we could hope to overcome this difficulty by the knowledge of extra information on the asymptotic behavior of $u_{n}$. The idea is to distinguish between the role of the a priori estimate supplied by $\mathscr{F}$ and the nondegeneracy condition, 
which should be satisfied only asymptotically by a suitable subset $\mathcal{U}(t)$ of the accumulation points of the sequences $\left\{u_{n}(t)\right\}_{n \in \mathbb{N}}$; this set is defined for a.e. $\left.t \in\right] 0, T[$ by

$$
\begin{aligned}
\mathcal{U}(t):=\left\{v \in A: \text { there exists an increasing subsequence } k \mapsto n_{k}\right. \text { s.t. } \\
\left.\qquad \lim _{k \uparrow+\infty} u_{n_{k}}(t)=v, \quad L v=\ell(t), \quad \limsup _{k \uparrow+\infty} \mathscr{F}\left(u_{n_{k}}(t)\right)<+\infty\right\} .
\end{aligned}
$$

By (P.2) we have

$$
\mathcal{U}(t) \subset D(\mathscr{F}) \text { for a.e. } t \in] 0, T[.
$$

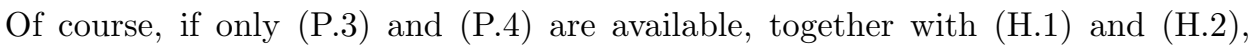
every point of $D(\mathscr{F})$ could belong to $\mathcal{U}(t)$ and $(\underline{\mathrm{H} .3})$ becomes necessary for the convergence, as we showed before.

However, we will show that (‥1) and $(\underline{\mathrm{H} .2})$ are sufficient to obtain some useful information on the pointwise asymptotic behavior of $u_{n}$ : roughly speaking, for a.e. $t \in] 0, T$ [ the sequence $\left\{u_{n}(t)\right\}_{n \in \mathbb{N}}$ becomes arbitrarily close to $\mathcal{U}(t)$ as $n \uparrow+\infty$. Any other information which could entail that $\mathcal{U}(t)$ is reduced to a single point for a.e. $t \in] 0, T\left[\right.$ would provide the desired strong convergence in $L^{q}(0, T ; A)$.

In order to measure the velocity of this asymptotic behavior, we recall the definition of the distance between a point $v \in A$ and a set $W \subseteq A$ :

$$
d(v, W):=\inf _{w \in W}\|v-w\|_{A}, \quad \text { with } \quad d(v, \varnothing)=+\infty .
$$

Theorem 2. Let (H.1) and (H.2) hold, let $u_{n} \in L^{q}(0, T ; A)$ be a sequence of functions satisfying (P.3) and (‥4), and let

$$
\mathcal{U}: t \in] 0, T[\mapsto \mathcal{U}(t) \subseteq A
$$

be the time-dependent multi-function whose values are the subsets of the accumulation points of $\left\{u_{n}(t)\right\}_{n \in \mathbb{N}}$ defined for a.e. $\left.t \in\right] 0, T[$ by (2.4). Then $\mathcal{U}(t)$ is nonempty for a.e. $t \in] 0, T\left[\right.$, the maps $t \mapsto \overline{\mathcal{U}(t)}$ and $t \mapsto d\left(u_{n}(t), \mathcal{U}(t)\right)$ are measurable, and

$$
\lim _{n \uparrow+\infty} \int_{0}^{T} d\left(u_{n}(t), \mathcal{U}(t)\right)^{q} d t=0,
$$

where $d$ is defined by (2.5).

Remark 2.4 (Measurability of $\mathcal{U}$ ). Since the functions $u_{n}$ are almost separably valued [Yos80, V.4], we can find a negligible set $N \subset] 0, T[$ such that

$$
A_{0}:=\overline{\operatorname{span}\left\{u_{n}(t): n \in \mathbb{N}, t \in\right] 0, T[\backslash N\}} \text { is separable and complete. }
$$

In particular,

$$
\left.\overline{\mathcal{U}(t)} \subset A_{0} \quad \forall t \in\right] 0, T[\backslash N,
$$

and therefore a (possible) definition of the measurability of the multi-function $\overline{\mathcal{U}}$ is CV77, Chap. III, §2, Thm.9]

$$
\text { the function } t \in] 0, T[\mapsto d(v, \mathcal{U}(t)) \text { is measurable } \forall v \in A \text {. }
$$

Corollary 1. Let (H.1) and (H.2) hold, let $u_{n} \in L^{q}(0, T ; A)$ be a sequence of functions satisfying ( $(\mathrm{P.3})$ and (P.4), and let $t \in] 0, T[\mapsto \mathcal{L}(t) \subset A$ denote the multifunction

$$
\left.\mathcal{L}(t):=L^{-1}(\ell(t)) \cap D(\mathscr{F}) \quad \text { for a.e. } t \in\right] 0, T[.
$$


Then

$$
\lim _{n \uparrow+\infty} \int_{0}^{T} d\left(u_{n}(t), \mathcal{L}(t)\right)^{q} d t=0 .
$$

Proof. (2.10) is an immediate consequence of (P.3) and (2.6), which imply

$$
\mathcal{U}(t) \subseteq \mathcal{L}(t) \quad \text { for a.e. } t \in] 0, T[.
$$

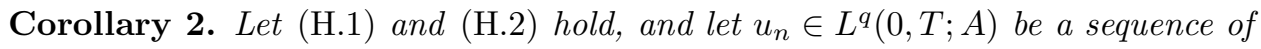
functions satisfying $(\mathbb{P . 3})$ and (․4 $)$. Then $u_{n}$ strongly converges in $L^{q}(0, T ; A)$ if and only if

$$
\mathcal{U}(t) \text { is nondegenerate w.r.t. } L \text { for a.e. } t \in] 0, T[\text {. }
$$

Proof. By (2.11),

$$
\mathcal{U}(t) \text { is a singleton } \Leftrightarrow \mathcal{U}(t) \text { is nondegenerate w.r.t. } L \text {. }
$$

Remark 2.5. Theorem 1 is an immediate consequence of the previous corollary: since $\mathcal{U}(t) \subset D(\mathscr{F})$ for a.e. $t \in] 0, T[$, (H.3) trivially implies (H.3').

Compactness for families of quasistationary problems. We have seen before that Theorems 1 and 2 are in some sense optimal, if we do not know any extra information on the sequence $\left\{u_{n}\right\}_{n \in \mathbb{N}}$; in particular, the nondegeneracy of $D(\mathscr{F})$ is an essential requirement for the convergence of $u_{n}$.

On the other hand, if we know that the functions $u_{n}$ satisfy suitable variational properties, it is possible to combine them with Theorem 2 in order to improve (2.6). Let us illustrate this feature by a simple example.

We consider another family of time-dependent functionals

$$
\left.\mathscr{G}_{n}:(t, v) \in\right] 0, T\left[\times A \rightarrow \mathscr{G}_{n}(t, v) \in[-\infty,+\infty], \quad n \in \mathbb{N},\right.
$$

which $\Gamma$-converges to $\mathscr{G}(t, \cdot)$ in $A$ for a.e. $t \in] 0, T[$ [Dal93, Chap. 4$]$ :

$$
\left.\mathscr{G}(t, \cdot)=\Gamma(A)-\lim _{n \uparrow+\infty} \mathscr{G}_{n}(t, \cdot) \quad \text { for a.e. } t \in\right] 0, T[.
$$

In order to measure "the degree of minimality" of $u_{n}(t)$ w.r.t. the functional $\mathscr{G}_{n}(t, \cdot)$, we introduce the quantity $\delta_{n}(t) \in[0,+\infty]$ defined by

$$
\left.\delta_{n}(t):=\sup _{v \in A}\left(\mathscr{G}_{n}\left(t, u_{n}(t)\right)-\mathscr{G}_{n}(t, v)\right) \quad \text { for a.e. } t \in\right] 0, T[;
$$

moreover, we will denote by $\mathcal{G}(t)$ the intersection of $\mathcal{L}(t)$ with the set of minimizers of $\mathscr{G}(t, \cdot)$ :

$$
\mathcal{G}(t):=\left\{v \in \mathcal{L}(t): \mathscr{G}(t, v)=\min _{w \in A} \mathscr{G}(t, w)\right\} .
$$

We have the following result.

Corollary 3. Let $\mathscr{F}, L, \mathscr{G}_{n}$ satisfy (P.1), (P.2), (2.13), (2.14); let $u_{n} \in L^{q}(0, T ; A)$ be a sequence satisfying (P.3), (P.4), and let $\delta_{n}$ be defined by (2.15). If

$$
\left.\lim _{n \uparrow+\infty} \delta_{n}(t)=0 \quad \text { a.e. in }\right] 0, T[\text {, }
$$


and (H.1), (H.2) hold with the nondegeneracy condition

$$
\mathcal{G}(t) \text { is nondegenerate w.r.t. } L \text { for a.e. } t \in] 0, T[\text {, }
$$

then $u_{n}$ strongly converges in $L^{q}(0, T ; A)$ as $n \uparrow+\infty$.

Proof. By the general theory of $\Gamma$-convergence [Dal93, Cor. 7.20], (2.14), (2.15), and (2.4) yield

$$
\mathcal{U}(t) \subseteq \mathcal{G}(t) \text { for a.e. } t \in] 0, T[.
$$

Applying Corollary 2 we obtain the desired conclusion.

Now we make a few remarks on the assumptions of the above results and on the possibility of some natural extensions.

Further extensions and remarks.

Recession functional and coercivity. We can give an alternative description of condition (H.2) by means of the notion of topological recession functional (see, e.g., [BBGT88]). For the sake of simplicity we are assuming that

$$
p=q=1, \quad A \text { is reflexive; }
$$

it is easy to modify the results when $q>1$ or when $A$ is the dual of a separable Banach space.

Definition 2.6. The weak sequential recession functional of $\mathscr{F}$ (w.r.t. $L$ ) is the functional defined by

$$
\mathscr{F}_{\infty}(v):=\inf \left\{\liminf _{n \uparrow+\infty} \lambda_{n}^{-1} \mathscr{F}\left(v_{0}+\lambda_{n} v_{n}\right): \lambda_{n} \uparrow+\infty, v_{n} \rightarrow v, L v_{n} \rightarrow L v\right\},
$$

where $v, v_{0} \in A$.

This definition is in fact independent of the choice of $v_{0}$ and gives rise to a positively homogeneous functional; in some sense, $\mathscr{F}_{\infty}(v)$ keeps track of the asymptotic behavior of $\mathscr{F}(w)$ as $w$ goes to infinity along the direction $v$.

Our interest in $\mathscr{F}_{\infty}$ lies in the following result, which shows that $(\underline{\mathrm{H} .2})$ is in fact equivalent to a sort of compatibility between $\mathscr{F}_{\infty}$ and $\operatorname{Ker} L$ :

Proposition 2.7. If (2.18) holds and $\mathcal{F}$ is compact in the sense of [BBGT88, (3.12)], then condition (स.21) is equivalent to

$$
v \in \operatorname{Ker} L \backslash\{0\} \quad \Rightarrow \quad \mathscr{F}_{\infty}(v)=+\infty .
$$

The lower semicontinuity of $\mathscr{F}$. We assumed that $\mathscr{F}$ is l.s.c. in order to simplify the exposition and to avoid subtle technicalities about measurability in (P.4).

Let us observe that it is always possible to replace $\mathscr{F}$ with its lower semicontinuous envelope

$$
\overline{\mathscr{F}}(v):=\inf \left\{\liminf _{k \uparrow+\infty} \mathscr{F}\left(v_{k}\right): v_{k} \rightarrow v\right\} \quad \forall v \in A,
$$

with proper domain

$$
\begin{array}{r}
D(\overline{\mathscr{F}}):=\left\{v \in A: \text { there exists a sequence } k \mapsto v_{k} \in A\right. \text { s.t. } \\
\left.\qquad \lim _{k \uparrow+\infty} v_{k}=v, \quad \sup _{k \in \mathbb{N}} \mathscr{F}\left(v_{k}\right)<+\infty\right\} .
\end{array}
$$


$\overline{\mathscr{F}}$ is the greatest lower semicontinuous real functional defined on $A$ that satisfies

$$
\overline{\mathscr{F}}(v) \leq \mathscr{F}(v) \quad \forall v \in A ;
$$

in particular, if the sequence $u_{n}$ satisfies (‥4) (where the measurability of $\mathscr{F}\left(u_{n}\right)$ is implicitly assumed), then the same estimate holds with $\overline{\mathscr{F}}$ instead of $\mathscr{F}$. It is then

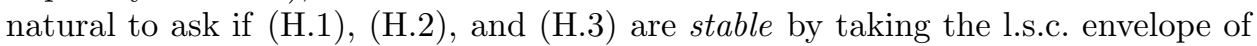
$\mathscr{F}$.

Proposition 2.8. If a proper functional $\mathscr{F}: A \rightarrow[0,+\infty]$ satisfies (H.1) and $(\underline{\mathrm{H} .2)}$, then also $\overline{\mathscr{F}}$ satisfies them; in particular, $(\overline{\mathrm{P} .2)}$ is not required for Theorem 2 and its Corollary 2 .

Remark 2.9. It is not difficult to see that we can replace (H.3) by

$$
D(\overline{\mathscr{F}}) \text { is nondegenerate, }
$$

if we want Problem $(\mathrm{P})$ to be always solvable for a generic proper functional $\mathscr{F}$.

Closed Operators. We could relax the continuity assumption (P.1) on $L$ as follows:

Theorems 1 and 2 hold for closed linear operators $L: D(L) \subseteq A \rightarrow B$.

In this case we intend that $u_{n}(t)$ belongs to the domain $D(L)$ of $L$ for a.e. $\left.t \in\right] 0, T$.

General measure spaces. The choice of functions defined on a time interval $] 0, T[$ and of the usual Lebesgue measure is irrelevant:

Theorems 1 and 2 hold for a general measure space $(I, \mathscr{M}, \mu), \mu$ being

a positive $\sigma$-additive measure with $\mu(I)<+\infty$ [Rud87, Chap. I].

\section{Proof of the Main Theorems}

\section{Two auxiliary results.}

Notation 3.1. For every $\zeta \in B$ and every sequence $\boldsymbol{v}:=\left\{v_{n}\right\}_{n \in \mathbb{N}}$ in $A$ we set

$$
\begin{aligned}
\Lambda_{\boldsymbol{v}, \zeta} & :=\{v \in D(\mathscr{F}): \text { there exists an increasing subsequence } \\
k & \left.\mapsto n_{k} \quad \text { s.t. } \quad \lim _{k \uparrow+\infty} v_{n_{k}}=v, \quad L v=\zeta, \quad \limsup _{k \uparrow+\infty} \mathscr{F}\left(v_{n_{k}}\right)<+\infty\right\} .
\end{aligned}
$$

Lemma 3.2. If (‥1) holds, then for every $q \in[1,+\infty[, w \in A, \zeta \in B$, and every sequence $\boldsymbol{v}:=\left\{v_{n}\right\}_{n \in \mathbb{N}}$ we have

$$
d\left(w, \Lambda_{\boldsymbol{v}, \zeta}\right)^{q}=\inf _{\varepsilon>0} \sup _{\sigma>0}\left\{\liminf _{n \uparrow+\infty}\left(\left\|w-v_{n}\right\|_{A}^{q}+\varepsilon \mathscr{F}\left(v_{n}\right)+\sigma\left\|L v_{n}-\zeta\right\|_{B}\right)\right\} .
$$

Proof. Let us first prove the inequality

$$
d\left(w, \Lambda_{\boldsymbol{v}, \zeta}\right)^{q} \geq \inf _{\varepsilon>0} \sup _{\sigma>0}\left\{\liminf _{n \uparrow+\infty}\left(\left\|w-v_{n}\right\|_{A}^{q}+\varepsilon \mathscr{F}\left(v_{n}\right)+\sigma\left\|L v_{n}-\zeta\right\|_{B}\right)\right\}
$$

in this case, it is not restrictive to assume that $\Lambda_{\boldsymbol{v}, \zeta} \neq \varnothing$. Therefore, we fix $v \in \Lambda_{\boldsymbol{v}, \zeta}$, $\varepsilon, \sigma>0$, and a subsequence $v_{n_{k}}$ as in (3.1), obtaining

$$
\begin{aligned}
& \liminf _{n \uparrow+\infty}\left(\left\|w-v_{n}\right\|_{A}^{q}+\varepsilon \mathscr{F}\left(v_{n}\right)+\sigma\left\|L v_{n}-\zeta\right\|_{B}\right) \\
& \leq \liminf _{k \uparrow+\infty}\left(\left\|w-v_{n_{k}}\right\|_{A}^{q}+\varepsilon \mathscr{F}\left(v_{n_{k}}\right)+\sigma\left\|L v_{n_{k}}-\zeta\right\|_{B}\right) \\
& \leq\|w-v\|_{A}^{q}+\varepsilon \limsup _{k \uparrow+\infty} \mathscr{F}\left(v_{n_{k}}\right) .
\end{aligned}
$$


Since $\varepsilon$ and $\sigma$ are arbitrary, we get

$$
\inf _{\varepsilon>0} \sup _{\sigma>0}\left\{\liminf _{n \uparrow+\infty}\left(\left\|w-v_{n}\right\|_{A}^{q}+\varepsilon \mathscr{F}\left(v_{n}\right)+\sigma\left\|L v_{n}-\zeta\right\|_{B}\right)\right\} \leq\|w-v\|_{A}^{q} .
$$

Finally, taking the infimum with respect to $v \in \Lambda_{\boldsymbol{v}, \zeta}$, we get 3.3 .

In order to prove the opposite inequality, for a fixed $\varepsilon>0$ let us denote by $s_{\varepsilon}$ the real number

$$
s_{\varepsilon}:=\sup _{\sigma>0}\left\{\liminf _{n \uparrow+\infty}\left(\left\|w-v_{n}\right\|_{A}^{q}+\varepsilon \mathscr{F}\left(v_{n}\right)+\sigma\left\|L v_{n}-\zeta\right\|_{B}\right)\right\}
$$

it is not restrictive to assume $s_{\varepsilon}<+\infty$.

By a standard diagonal argument, there exists an increasing sequence $k \mapsto n_{k} \in$ $\mathbb{N}$ such that

$$
s_{\varepsilon}=\lim _{k \uparrow+\infty}\left(\left\|w-v_{n_{k}}\right\|_{A}^{q}+\varepsilon \mathscr{F}\left(v_{n_{k}}\right)+k\left\|L v_{n_{k}}-\zeta\right\|_{B}\right)
$$

in particular,

$$
\limsup _{k \uparrow+\infty} \mathscr{F}\left(v_{n_{k}}\right)<+\infty, \quad \lim _{k \uparrow+\infty} L v_{n_{k}}=\zeta
$$

By the compactness assumption H.1 we can extract a further subsequence, say $v_{n_{k}^{\prime}}$, such that

$$
\lim _{k \uparrow+\infty} v_{n_{k}^{\prime}}=v_{\infty} \in \Lambda_{\boldsymbol{v}, \zeta}, \quad\left\|w-v_{\infty}\right\|_{A}=\liminf _{k \uparrow+\infty}\left\|w-v_{n_{k}}\right\|_{A}
$$

Therefore, we have

$$
d\left(w, \Lambda_{\boldsymbol{v}, \zeta}\right)^{q} \leq\left\|w-v_{\infty}\right\|_{A}^{q} \leq \liminf _{k \uparrow+\infty}\left\|w-v_{n_{k}}\right\|_{A}^{q} \leq s_{\varepsilon} .
$$

Since $\varepsilon$ is arbitrary, the proof is finished.

Lemma 3.3. If (H.1) and (H.2) hold, then for every $\zeta \in B$ and every sequence $\boldsymbol{v}:=\left\{v_{n}\right\}_{n \in \mathbb{N}}$ in $A$ such that the set $\Lambda_{\boldsymbol{v}, \zeta}$ defined by (3.1) is not empty, we have

(3.4) $\forall \varepsilon>0 \exists C_{\varepsilon}>0: d\left(v_{n}, \Lambda_{\boldsymbol{v}, \zeta}\right)^{q} \leq \varepsilon\left(1+\mathscr{F}\left(v_{n}\right)\right)+C_{\varepsilon}\left\|L v_{n}-\zeta\right\|_{B}^{p} \quad \forall n \in \mathbb{N}$.

Proof. Let us argue by contradiction. Suppose (3.4) is not true; then we could find an $\varepsilon_{0}>0$ and an increasing subsequence $k \mapsto n_{k}$ s.t.

$$
d\left(v_{n_{k}}, \Lambda_{\boldsymbol{v}, \zeta}\right)^{q} \geq \varepsilon_{0}\left(1+\mathscr{F}\left(v_{n_{k}}\right)\right)+k\left\|L v_{n_{k}}-\zeta\right\|_{B}^{p} \quad \forall k \in \mathbb{N} .
$$

We distinguish two cases:

- $v_{n_{k}}$ is bounded in $A$ : in this case

$$
\sup _{k \in \mathbb{N}} \mathscr{F}\left(v_{n_{k}}\right)<+\infty, \quad \lim _{k \uparrow+\infty}\left\|L v_{n_{k}}-\zeta\right\|_{B}=0
$$

so that by (H.1) $k \mapsto v_{n_{k}}$ has at least one accumulation point, which a fortiori belongs to $\Lambda_{\boldsymbol{v}, \zeta}$; this contradicts (3.5), which forces $d\left(v_{n_{k}}, \Lambda_{\boldsymbol{v}, \zeta}\right)$ to be greater than $\varepsilon_{0}>0$.

- $v_{n_{k}}$ is unbounded in $A$ : we fix $v_{\infty} \in \Lambda_{\boldsymbol{v}, \zeta}$, and we observe that

$$
\left\|v_{n_{k}}\right\|_{A}^{q} \geq \frac{1}{2}\left\|v_{n_{k}}\right\|_{A}^{q}+\frac{1}{2^{q}} d\left(v_{n_{k}}, \Lambda_{\boldsymbol{v}, \zeta}\right)^{q}-\frac{1}{2}\left\|v_{\infty}\right\|_{A}^{q} .
$$

By (3.5) we obtain

$$
\begin{aligned}
\left\|v_{n_{k}}\right\|_{A}^{q} & \geq \frac{1}{2}\left\|v_{n_{k}}\right\|_{A}^{q}+\frac{\varepsilon_{0}}{2^{q}} \mathscr{F}\left(v_{n_{k}}\right)+\frac{k}{2^{q}}\left\|L v_{n_{k}}-\zeta\right\|_{B}^{p}-\frac{1}{2}\left\|v_{\infty}\right\|_{A}^{q} \\
& \geq \frac{\varepsilon_{0}}{2^{q}} \mathscr{F}\left(v_{n_{k}}\right)+\rho_{k}\left(1+\left\|L v_{n_{k}}\right\|_{B}^{p}\right),
\end{aligned}
$$


where

$$
\rho_{k}:=\frac{1}{2}\left(1+\left\|L v_{n_{k}}\right\|_{B}^{p}\right)^{-1}\left(\left\|v_{n_{k}}\right\|_{A}^{q}+\frac{k}{2^{q-1}}\left\|L v_{n_{k}}-\zeta\right\|_{B}^{p}-\left\|v_{\infty}\right\|_{A}^{q}\right) .
$$

(3.6) contradicts (H.2) since lim $\sup _{k \uparrow+\infty} \rho_{k}=+\infty$.

\section{Proof of Theorem 2 ,}

Preliminaries. Now we can prove Theorem 2 in the case of a general measure space $(I, \mathscr{M}, \mu)$ as in section 2, therefore, we will assume that $u_{n}$ is a sequence of functions in $L^{q}(I ; A)$ with

$$
\exists \ell:=\lim _{n \uparrow+\infty} L u_{n} \quad \text { in } L^{p}(I ; B) \quad \text { for some } p \in[1,+\infty),
$$

and

$$
S:=\sup _{n \in \mathbb{N}} \int_{I} \mathscr{F}\left(u_{n}(t)\right) d \mu(t)<+\infty ;
$$

(‥2) yields in particular that

$$
S^{\prime}:=\sup _{n \in \mathbb{N}} \int_{I}\left\|u_{n}(t)\right\|_{A}^{q} d \mu(t)<+\infty .
$$

Since the functions $u_{n}$ are $\mu$-almost separably valued [Yos80, V.4], it is not restrictive to assume that

$A$ is separable.

Claim 1. $\mathcal{U}(t) \neq \varnothing$ for $\mu$-a.e. $t \in I$. Thanks to (3.7), there exists an increasing sequence $k \mapsto n_{k}$ such that

$$
\lim _{k \uparrow+\infty} L u_{n_{k}}(t)=\ell(t) \quad \text { strongly in } B \text {, for } \mu \text {-a.e. } t \in I \text {. }
$$

(3.8) and Fatou's Lemma yield

$$
\int_{I}\left(\liminf _{k \uparrow+\infty} \mathscr{F}\left(u_{n_{k}}(t)\right)\right) d t \leq S<+\infty
$$

and, in particular,

$$
\liminf _{k \uparrow+\infty} \mathscr{F}\left(u_{n_{k}}(t)\right)<+\infty \quad \text { for } \mu \text {-a.e. } t \in I .
$$

Taking account of the conditional compactness assumption (H.1), we deduce that the sets $\mathcal{U}(t)$ defined by (2.4) are not empty for every $t$ in a measurable subset $I^{\prime} \subseteq I$ with $\mu\left(I \backslash I^{\prime}\right)=0$.

Claim 2. If $t \in I \mapsto w(t) \in A$ is (strongly) measurable, then the map

$$
t \in I \mapsto d(w(t), \mathcal{U}(t))
$$

is measurable. This is a simple consequence of Lemma 3.2 since for every $t \in I^{\prime}$,

$$
\mathcal{U}(t)=\Lambda_{\left\{u_{n}(t)\right\}, \ell(t)} ;
$$

in particular,

$$
\begin{aligned}
& d(w(t), \mathcal{U}(t)) \\
& =\inf _{\varepsilon>0} \sup _{\sigma>0}\left\{\liminf _{n \uparrow+\infty}\left(\left\|w(t)-u_{n}(t)\right\|_{A}+\varepsilon \mathscr{F}\left(u_{n}(t)\right)+\sigma\left\|L u_{n}(t)-\ell(t)\right\|_{B}\right)\right\} .
\end{aligned}
$$


Observe that this property implies the measurability of the multifunction $t \mapsto \overline{\mathcal{U}(t)}$ (see Remark 2.4).

Claim 3. The functions $t \in I \mapsto\left\|u_{n}(t)\right\|_{A}^{q}$ are equi-integrable.

Since $L u_{n} \rightarrow \ell$ in $L^{p}(I ; B)$, it is easy to see that

$$
\lim _{\mu(J) \downarrow 0} \int_{J}\left\|L u_{n}(t)\right\|_{B}^{p} d \mu(t)=0 \quad \text { uniformly w.r.t. } n \text {. }
$$

Therefore, by (H.2),

$$
\begin{aligned}
\limsup _{\mu(J) \downarrow 0} \int_{J}\left\|u_{n}(t)\right\|_{A}^{q} d \mu(t) & \leq \varepsilon\left(\sup _{n} \int_{I} \mathscr{F}\left(u_{n}(t)\right) d \mu(t)\right) \\
& +C_{\varepsilon} \limsup _{\mu(J) \downarrow 0} \int_{J}\left(1+\left\|L u_{n}(t)\right\|_{B}^{p}\right) d \mu(t) \leq \varepsilon S .
\end{aligned}
$$

Since $\varepsilon$ is arbitrary, we deduce that

$$
\lim _{\mu(J) \downarrow 0} \int_{J}\left\|u_{n}(t)\right\|_{A}^{q} d \mu(t)=0 \quad \text { uniformly w.r.t. } n .
$$

Claim 4. The functions $t \mapsto d\left(u_{n}(t), \mathcal{U}(t)\right)^{q}$ are equi-integrable.

We observe that

$$
d\left(u_{n}(t), \mathcal{U}(t)\right)^{q} \leq 2^{q-1}\left(\left\|u_{n}(t)\right\|_{A}^{q}+d(0, \mathcal{U}(t))^{q}\right)
$$

taking account of the previous claim, it will be sufficient to prove that

$$
\int_{I} d(0, \mathcal{U}(t))^{q} d \mu(t)<+\infty .
$$

Starting from (3.2) and recalling (3.7), (3.8), and (3.9), we see by Beppo Levi's Theorem and Fatou's Lemma that

$$
\begin{aligned}
& \int_{I} d(0, \mathcal{U}(t))^{q} d \mu(t) \\
& \leq \int_{I} \lim _{\sigma \uparrow+\infty} \liminf _{n \uparrow+\infty}\left(\left\|u_{n}(t)\right\|_{A}^{q}+\mathscr{F}\left(u_{n}(t)\right)+\sigma\left\|L u_{n}(t)-\ell(t)\right\|_{B}\right) d \mu(t) \\
& =\lim _{\sigma \uparrow+\infty} \int_{I} \liminf _{n \uparrow+\infty}\left(\left\|u_{n}(t)\right\|_{A}^{q}+\mathscr{F}\left(u_{n}(t)\right)+\sigma\left\|L u_{n}(t)-\ell(t)\right\|_{B}\right) d \mu(t) \\
& \leq \limsup _{\sigma \uparrow+\infty}\left(S^{\prime}+S+\sigma \lim _{n \uparrow+\infty} \int_{I}\left\|L u_{n}(t)-\ell(t)\right\|_{B} d \mu(t)\right) \leq S^{\prime}+S<+\infty .
\end{aligned}
$$

Claim 5. $\lim _{n \uparrow+\infty} \int_{I} d\left(u_{n}(t), \mathcal{U}(t)\right)^{q} d \mu(t)=0$.

For fixed $\varepsilon, M>0$ we denote by $I_{\varepsilon, M}$ the subset of $I$

$$
\begin{aligned}
I_{\varepsilon, M}:=\{t & \in I^{\prime}: d\left(u_{n}(t), \mathcal{U}(t)\right)^{q} \\
& \left.\leq \varepsilon\left(1+\mathscr{F}\left(u_{n}(t)\right)\right)+M\left\|L u_{n}(t)-\ell(t)\right\|_{B}^{p} \quad \forall n \in \mathbb{N} .\right\} .
\end{aligned}
$$

It is obvious that

$$
\varepsilon \leq \varepsilon^{\prime}, \quad M \leq M^{\prime} \Rightarrow I_{\varepsilon, M} \subseteq I_{\varepsilon^{\prime}, M^{\prime}} .
$$

By Lemma 3.3 we know that

$$
\bigcup_{M>0} I_{\varepsilon, M}=I^{\prime}, \quad \forall \varepsilon>0 ;
$$


therefore,

$$
\lim _{M \uparrow+\infty} \mu\left(I \backslash I_{\varepsilon, M}\right)=0 .
$$

We obtain

$$
\begin{aligned}
\limsup _{n \uparrow+\infty} & \int_{I} d\left(u_{n}(t), \mathcal{U}(t)\right)^{q} d \mu(t) \leq \limsup _{n \uparrow+\infty} \int_{I \backslash I_{\varepsilon, M}} d\left(u_{n}(t), \mathcal{U}(t)\right)^{q} d \mu(t) \\
& +\varepsilon(1+S)+M \limsup _{n \uparrow+\infty} \int_{I_{\varepsilon, M}}\left\|L u_{n}(t)-\ell(t)\right\|^{p} d \mu(t) \\
& \leq \limsup _{n \uparrow+\infty} \int_{I \backslash I_{\varepsilon, M}} d\left(u_{n}(t), \mathcal{U}(t)\right)^{q} d \mu(t)+\varepsilon(1+S) .
\end{aligned}
$$

When $M \uparrow+\infty$, by the previous claim and (3.12), we get

$$
\limsup _{n \uparrow+\infty} \int_{I} d\left(u_{n}(t), \mathcal{U}(t)\right)^{q} d \mu(t) \leq \varepsilon(1+S) .
$$

Finally, we choose $\varepsilon$ arbitrarily small and conclude the proof.

Proof of Proposition 2.7. Let us first prove that (H.2) implies ( implication does not require $A$ to be reflexive.

We fix

$$
v_{0} \in A, \quad v \in \operatorname{Ker} L, \quad v \neq 0,
$$

and two sequences $n \mapsto \lambda_{n} \in \mathbb{R}, n \mapsto v_{n} \in A$ such that

$$
\lambda_{n} \uparrow+\infty, \quad v_{n} \rightarrow v, \quad L v_{n} \rightarrow L v \quad \text { as } n \uparrow+\infty .
$$

Thanks to (H.2) we obtain

$$
\varepsilon \mathscr{F}\left(v_{0}+\lambda_{n} v_{n}\right) \geq \lambda_{n}\left\|v_{n}\right\|_{A}-\left\|v_{0}\right\|_{A}-C_{\varepsilon}\left(1+\left\|L v_{0}\right\|_{B}+\lambda_{n}\left\|L v_{n}\right\|_{B}\right),
$$

and, dividing both sides by $\lambda_{n}$,

$$
\varepsilon \lambda_{n}^{-1} \mathscr{F}\left(v_{0}+\lambda_{n} v_{n}\right) \geq\left\|v_{n}\right\|_{A}-C_{\varepsilon}\left\|L v_{n}\right\|_{B}-\lambda_{n}^{-1}\left(\left\|v_{0}\right\|_{A}+C_{\varepsilon}\left(1+\left\|L v_{0}\right\|_{B}\right)\right) .
$$

Passing to the limit as $n \uparrow+\infty$ and taking into account (3.13) and

$$
\liminf _{n \uparrow+\infty}\left\|v_{n}\right\|_{A} \geq\|v\|_{A}>0, \quad \lim _{n \uparrow+\infty}\left\|L v_{n}\right\|_{B}=\|L v\|_{B}=0
$$

we get

$$
\liminf _{n \uparrow+\infty} \lambda_{n}^{-1} \mathscr{F}\left(v_{0}+\lambda_{n} v_{n}\right) \geq \varepsilon^{-1}\|v\|_{A} .
$$

Since $\varepsilon$ can be chosen arbitrarily small, we deduce $\left(\underline{H} .2^{\prime}\right)$.

Now let us prove the opposite implication; we argue by contradiction, and we suppose that there exist $\varepsilon>0$ and a sequence $\left\{w_{n}\right\}_{n \in \mathbb{N}}$ in $A$ such that

$$
\left\|w_{n}\right\|_{A} \geq \varepsilon \mathscr{F}\left(w_{n}\right)+n\left(1+\left\|L w_{n}\right\|_{B}\right) .
$$

We set $\lambda_{n}:=\left\|w_{n}\right\|_{A}, v_{n}:=\lambda_{n}^{-1} w_{n}$, and we observe that (3.14) yields

$$
\lim _{n \uparrow+\infty} \lambda_{n}=+\infty, \quad \varepsilon \lambda_{n}^{-1} \mathscr{F}\left(\lambda_{n} v_{n}\right)+n\left\|L v_{n}\right\| \leq 1 .
$$

Therefore, we can extract a subsequence (still denoted by $v_{n}$ ) such that

$$
v_{n} \rightarrow v, \quad L v_{n} \rightarrow 0=L v \quad \text { as } n \uparrow+\infty ;
$$


by Definition 2.6 of the recession functional and the compactness property BBGT88, (3.12)] we get

$$
\varepsilon \mathscr{F}_{\infty}(v) \leq 1, v \neq 0,
$$

which contradicts $\left(\underline{\mathrm{H} .2^{\prime}}\right)$ since $v \in \operatorname{Ker} L$.

Proof of Proposition 2.8, Let us first check (H.1): we take a sequence $n \mapsto v_{n}$ such that

$$
L v_{n} \rightarrow \ell \in B, \quad \sup _{n \in \mathbb{N}} \overline{\mathscr{F}}\left(v_{n}\right)=s<+\infty .
$$

By the definition (2.19) of $\overline{\mathscr{F}}$, there exists another sequence $n \mapsto w_{n} \in D(\mathscr{F})$ such that

$$
\lim _{n \uparrow+\infty}\left\|w_{n}-v_{n}\right\|_{A}=0, \quad \sup _{n \in \mathbb{N}} \mathscr{F}\left(w_{n}\right) \leq s+1 .
$$

By (3.16) and the first limit of (3.17), $L w_{n} \rightarrow \ell$ in $B$. Therefore, by (H.1), we can find a subsequence $k \mapsto w_{n_{k}}$ strongly converging in $A$; (3.17) yields the convergence of $k \mapsto v_{n_{k}}$.

In order to check that $\overline{\mathscr{F}}$ satisfies $(\underline{\mathrm{H} .2})$, it is sufficient to notice that the map

$$
v \mapsto\|v\|_{A}^{q}-C_{\varepsilon}\left(1+\|L v\|_{B}^{p}\right)
$$

is continuous in $A$ and is bounded from above by $\varepsilon \mathscr{F}$; by the extremal property of $\overline{\mathscr{F}},(3.18)$ is less than $\varepsilon \overline{\mathscr{F}}$, too.

Proof of Theorem 2 for closed operators. We replace $A$ with the Banach space $D(L)$ endowed with the graph norm

$$
\|v\|_{D(L)}:=\|v\|_{A}+\|L v\|_{B},
$$

which makes $L$ continuous; we will denote by $\mathscr{F}$ again its restriction to $D(L)$, and we simply have to check that $(\underline{\mathrm{H} .1})$ and $(\underline{\mathrm{H} .2})$ are satisfied in this setting, too.

Since a sequence $\left\{v_{n}\right\}$ in $D(L)$ converges in $D(L)$ iff it converges in $A$ and $L v_{n}$ converges in $B$, $($ H.1) is immediate; $(\underline{H .2})$ is also obvious, since the $L$-part of the norm of $D(L)$ is already controlled by the right-hand side of (ㅍ.2).

\section{Applications}

An abstract version of the Luckhaus Theorem. First of all, we will consider the situation of Example 2, so we are assuming that

$$
B \text { is a Banach space, } \mathscr{F}_{1}, \mathscr{F}_{2}: B \rightarrow[0,+\infty] \text { are proper l.s.c. functionals. }
$$

Theorem 3. Let $1 \leq q \leq p<+\infty$ and let $\left.\theta_{n}, \chi_{n}:\right] 0, T[\rightarrow B$ be two sequences of (strongly) measurable functions satisfying

$$
\int_{0}^{T} \mathscr{F}_{1}\left(\theta_{n}(t)\right) d t+\int_{0}^{T} \mathscr{F}_{2}\left(\chi_{n}(t)\right) d t \leq S<+\infty \quad \forall n \in \mathbb{N},
$$

and

$$
\theta_{n}+\chi_{n} \rightarrow \ell \text { strongly in } L^{p}(0, T ; B) .
$$


the sublevels of $\mathscr{F}_{1}$ or $\mathscr{F}_{2}$ are compact in $B$,

then, denoting

$$
\lim _{\|v\|_{B} \uparrow+\infty} \frac{\mathscr{F}_{1}(v)}{\|v\|_{B}^{q}}=+\infty \quad \text { or } \quad \lim _{\|v\|_{B} \uparrow+\infty} \frac{\mathscr{F}_{2}(v)}{\|v\|_{B}^{q}}=+\infty,
$$

$$
\mathcal{L}(t):=\left\{(\theta, \chi) \in D\left(\mathscr{F}_{1}\right) \times D\left(\mathscr{F}_{2}\right): \theta+\chi=\ell(t)\right\},
$$

we have

$$
\lim _{n \uparrow+\infty} \int_{0}^{T} \inf _{(\theta, \chi) \in \mathcal{L}(t)}\left\{\left\|\theta_{n}(t)-\theta\right\|_{B}^{q}+\left\|\chi_{n}(t)-\chi\right\|_{B}^{q}\right\} d t=0 .
$$

In particular, if $\mathscr{F}_{1}, \mathscr{F}_{2}$ satisfy

$$
\left.\begin{array}{c}
\theta, \theta^{\prime} \in D\left(\mathscr{F}_{1}\right), \quad \chi, \chi^{\prime} \in D\left(\mathscr{F}_{2}\right) \\
\theta+\chi=\theta^{\prime}+\chi^{\prime}
\end{array}\right\} \Rightarrow \theta=\theta^{\prime}, \quad \chi=\chi^{\prime},
$$

then

$$
\exists \theta, \chi \in L^{q}(0, T ; B): \quad \theta_{n} \rightarrow \theta, \quad \chi_{n} \rightarrow \chi \quad \text { strongly in } L^{q}(0, T, B) .
$$

Proof. As we did in Example2, we set

$$
\begin{gathered}
A:=B \times B, \quad\|(\theta, \chi)\|_{A}:=\max \left(\|\theta\|_{B},\|\chi\|_{B}\right), \quad L: u=(\theta, \chi) \rightarrow \theta+\chi, \\
\mathscr{F}(u):=\mathscr{F}_{1}(\theta)+\mathscr{F}_{2}(\chi),
\end{gathered}
$$

and we apply Theorem 2 with its corollary; therefore, we have to check that (H.1) and (H.2) are satisfied.

Control of H.1. We take a sequence

$$
u_{n}:=\left(\theta_{n}, \chi_{n}\right) \in A \text { such that } \theta_{n}+\chi_{n} \rightarrow \zeta \text { in } B
$$

and

$$
\mathscr{F}_{1}\left(\theta_{n}\right)+\mathscr{F}_{2}\left(\chi_{n}\right) \leq s<+\infty \quad \forall n \in \mathbb{N} .
$$

Let us suppose that in (4.2), e.g., the sublevels of $\mathscr{F}_{1}$ are compact; then we can extract a subsequence $k \mapsto \theta_{n_{k}}$, converging in $B$, so that (4.9) yields the convergence of $\chi_{n_{k}}$ in $B$ and $u_{n_{k}}$ in $A$, too.

Control of (H.2). We can suppose that the first limit of (4.3) holds; it is easy to see that it is equivalent to

$$
\forall \varepsilon>0 \exists c_{\varepsilon} \geq 0: \quad\|\theta\|_{B}^{q} \leq \varepsilon \mathscr{F}_{1}(\theta)+c_{\varepsilon} \quad \forall \theta \in B .
$$

Therefore, we get

$$
\begin{aligned}
\|u\|_{A}^{q} & \leq \max \left(\|\theta\|_{B}^{q},\|\chi\|_{B}^{q}\right) \leq 2^{q-1}\left(\|\theta\|_{B}^{q}+\|\theta+\chi\|_{B}^{q}\right) \\
& \leq 2^{q-1}\left(\varepsilon \mathscr{F}_{1}(\theta)+2 c_{\varepsilon}+\|\theta+\chi\|_{B}^{p}\right) \leq 2^{q-1}\left(\varepsilon \mathscr{F}(u)+c_{\varepsilon}+\|L u\|_{B}^{p}\right),
\end{aligned}
$$

so that (H.2) is satisfied. In order to prove that (4.5) implies (4.6), we apply Theorem 1 and we observe that (4.5) is equivalent to (H.3) in the framework of (4.7), (4.8).

If we particularize the choice of $\mathscr{F}_{1}$ and $\mathscr{F}_{2}$ we easily get a refinement of the Luckhaus and Plotnikov Theorems. 
Corollary 4. In the framework of (1.10),,$\ldots$, (1.14), (1.18), with $1 \leq q \leq p<+\infty$, let us assume that one of the following conditions holds:

i. $K$ is compact in $B$;

ii. $K$ is bounded and the inclusion of $V$ in $B$ is compact;

iii. the inclusion of $V$ in $B$ is compact and $q<p$.

Then, denoting

$$
\mathcal{L}(t):=\{(\theta, \chi) \in V \times K: \theta+\chi=\ell(t)\},
$$

we have

$$
\lim _{n \uparrow+\infty} \int_{0}^{T} \inf _{(\theta, \chi) \in \mathcal{L}(t)}\left\{\left\|\theta_{n}(t)-\theta\right\|_{B}^{q}+\left\|\chi_{n}(t)-\chi\right\|_{B}^{q}\right\} d t=0 .
$$

In particular, if (1.15) holds too, then

$$
\exists \theta, \chi \in L^{q}(0, T ; B): \quad \theta_{n} \rightarrow \theta, \quad \chi_{n} \rightarrow \chi \quad \text { strongly in } L^{q}(0, T, B) .
$$

Proof. It is sufficient to apply Theorem 3 with $\mathscr{F}_{1}, \mathscr{F}_{2}$ given by (1.17).

Remark 4.1. It is easy to obtain a simple variant of the previous corollary in the case of a time-dependent family of closed sets $K(t)$ (see [HS98a, HS98b]): if (1.12) is replaced by

$$
\left.\theta_{n}(t) \in V, \quad \chi_{n}(t) \in K(t) \subset K \quad \text { for a.e. } t \in\right] 0, T[,
$$

where $\{K(t)\}_{t \in] 0, T[}$ is a family of closed nonempty sets that satisfy

$$
\left.\chi, \chi^{\prime} \in K(t), \quad \chi-\chi^{\prime} \in V \quad \Rightarrow \quad \chi=\chi^{\prime} \quad \text { for a.e. } t \in\right] 0, T[,
$$

then we can deduce (4.13), under the same other assumptions of the previous corollary. It is sufficient to apply Corollary 2, taking into account that in this case

$$
\mathcal{U}(t) \subseteq V \times K(t) \text { for a.e. } t \in] 0, T[.
$$

Quasistationary phase field equations with Neumann boundary conditions. In this last subsection, we will apply Corollary 3 to refine the proof of Sch00 about the convergence of solutions of quasistationary phase field equations with Neumann boundary conditions to solutions of the Stefan problem with the Gibbs-Thomson law.

Following Sch00, we define $\Omega, Q$ as in (1.1), $W: s \in \mathbb{R} \mapsto\left(s^{2}-1\right)^{2}, \varepsilon>0$,

$$
G_{\varepsilon}:(\chi, w) \in H^{1}(\Omega) \times L^{2}(\Omega) \mapsto \int_{\Omega}\left(\varepsilon|\nabla \chi|^{2}+\frac{1}{\varepsilon} W(\chi)+\frac{1}{2} \chi^{2}-w \chi\right) d x .
$$

For $f \in L^{2}(Q), w \in L^{2}(\Omega), \delta_{\varepsilon}>0$, we look for the functions $\theta_{\varepsilon}, \chi_{\varepsilon}, w_{\varepsilon}$, which solve

$$
\begin{gathered}
w_{\varepsilon}=\chi_{\varepsilon}+\theta_{\varepsilon}, \\
\begin{cases}\partial_{t} w_{\varepsilon}-\Delta \theta_{\varepsilon}=f & \text { in } Q, \\
\partial_{\nu} \theta_{\varepsilon}=0 & \text { on } \partial \Omega \times] 0, T[, \\
w_{\varepsilon}(x, 0)=\underline{w}(x) & \text { in } \Omega ;\end{cases} \\
\begin{cases}-2 \varepsilon \Delta \chi_{\varepsilon}+\frac{1}{\varepsilon} W^{\prime}\left(\chi_{\varepsilon}\right)=\theta_{\varepsilon} & \text { in } Q, \\
\partial_{\nu} \chi_{\varepsilon}=0 & \text { on } \partial \Omega \times] 0, T[;\end{cases} \\
\left.G_{\varepsilon}\left(\chi_{\varepsilon}(\cdot, t), w_{\varepsilon}(\cdot, t)\right) \leq G_{\varepsilon}\left(v, w_{\varepsilon}(\cdot, t)\right)+\delta_{\varepsilon} \quad \forall v \in H^{1}(\Omega), \text { a.e. in }\right] 0, T[.
\end{gathered}
$$


Sch00, Theorem 2.2] provides the existence of $\left(\theta_{\varepsilon}, \chi_{\varepsilon}, w_{\varepsilon}\right)$ for every $\varepsilon>0$, together with $\delta_{\varepsilon} \downarrow 0$ as $\varepsilon$ goes to 0 , the a priori estimates

$$
\begin{gathered}
\left\|\partial_{t} w_{\varepsilon}\right\|_{L^{2}\left(0, T ; H^{-1}(\Omega)\right)},\left\|\theta_{\varepsilon}\right\|_{L^{2}\left(0, T ; H^{1}(\Omega)\right)} \leq S, \\
\left.\int_{\Omega}\left(\left|\chi_{\varepsilon}(x, t)\right|^{4}+\left|\nabla \chi_{\varepsilon}(x, t)\right|\right) d x \leq S \quad \text { for a.e. } t \in\right] 0, T[,
\end{gathered}
$$

and the compactness property

$$
\exists \varepsilon_{n} \downarrow 0: \quad w_{\varepsilon_{n}} \rightarrow w \quad \text { strongly in } L^{2}(Q) \text { and a.e. in } Q .
$$

Let us recall Giu84 that $B V(\Omega)$ denotes the Banach space of the functions $v \in$ $L^{1}(\Omega)$ with finite total variation

$$
\int_{\Omega}|D v|:=\sup \left\{\int_{\Omega} v(x) \operatorname{div} \phi(x) d x: \phi \in C_{0}^{\infty}\left(\Omega ; \mathbb{R}^{N}\right),|\phi(x)| \leq 1 \text { in } \Omega\right\}<+\infty .
$$

We also introduce the subset of $B V(\Omega)$,

$$
B V(\Omega ;\{-1,1\}):=\{v \in B V(\Omega):|v(x)|=1 \text { for a.e. } x \in \Omega\},
$$

and we can prove the following result:

Theorem 4. Let us assume that the function $\rho:[0, T] \rightarrow \mathbb{R}$, defined by

$$
\rho(s):=\int_{\Omega} \underline{w}(x) d x+\int_{0}^{s} \int_{\Omega} f(x, t) d x d t,
$$

satisfies

$$
\rho(s) \neq 0 \quad \text { for a.e. } s \in] 0, T[\text {. }
$$

Then the limits

$$
\lim _{n \rightarrow+\infty} \theta_{\varepsilon_{n}}=\theta, \quad \lim _{n \rightarrow+\infty} \chi_{\varepsilon_{n}}=\chi \quad \text { strongly in } L^{2}(Q)
$$

exist, and they are solutions of the quasistationary Stefan problem with Neumann boundary condition

$$
\begin{gathered}
w=\chi+\theta, \\
\begin{cases}\partial_{t} w-\Delta \theta=f & \text { in } Q, \\
\partial_{\nu} \theta=0 & \text { on } \partial \Omega \times] 0, T[, \\
w(x, 0)=\underline{w}(x) & \text { in } \Omega ;\end{cases} \\
x \mapsto \chi(t, x) \in B V(\Omega ;\{-1,1\}) \quad \text { for a.e. } t \in] 0, T[, \\
G(\chi(\cdot, t), w(\cdot, t)) \leq G(v, w(\cdot, t)) \quad \forall v \in B V(\Omega ;\{-1,1\}),
\end{gathered}
$$

where

$$
G(v, w):= \begin{cases}\frac{4}{3} \int_{\Omega}|D v|-\int_{\Omega} w(x) v(x) d x & \text { if } v \in B V(\Omega ;\{-1,1\}) \\ +\infty & \text { otherwise. }\end{cases}
$$

Proof. The theorem follows by standard convergence arguments if we prove (4.23). Let us denote by $I^{\prime}$ the subset of the points $\left.t \in\right] 0, T[$ where the sequence $n \mapsto$ $w_{\varepsilon_{n}}(\cdot, t)$ converges in $L^{2}(\Omega)$ and $\rho(t) \neq 0$; by (4.22) $] 0, T\left[\backslash I^{\prime}\right.$ is negligible. 
We apply Corollary 3 with $p=q=2$ and

$$
\begin{gathered}
B:=L^{2}(\Omega), \quad A:=B \times B, \quad L: u=(\theta, \chi) \mapsto \theta+\chi \\
\mathscr{F}(u):=\int_{\Omega}|\theta(x)|^{2}+|\nabla \theta(x)|^{2}+|\chi(x)|^{4} d x, \\
\mathscr{G}_{n}(t, u):=G_{\varepsilon_{n}}\left(\chi, w_{\varepsilon_{n}}(\cdot, t)\right) \quad \text { for } u=(\theta, \chi),
\end{gathered}
$$

where we adopted the usual convention of setting $\mathscr{F}(u)=+\infty$ if $\theta \notin H^{1}(\Omega)$ or $\chi \notin L^{4}(\Omega)$, and $\mathscr{G}_{n}(t, u)=+\infty$ if $\chi \notin H^{1}(\Omega)$. Therefore, we are in the situation of Problem (P), and, arguing as in Theorem 3 and its corollary, we can easily check (H.1) and (‥2).

In order to complete the argument, we have to study the minimum set $\mathcal{G}(t)$ of the $\Gamma\left(L^{2}(\Omega)\right)$-limit $\mathscr{G}$ of $\mathscr{G}_{n}$. The result of [Mod86] and a standard perturbation argument Dal93] show that this functional is

$$
\mathscr{G}(t, u):=G(\chi, w(\cdot, t))+\frac{1}{2}|\Omega| \quad \forall t \in I^{\prime} .
$$

If $u_{i}:=\left(\theta_{i}, \chi_{i}\right), i=1,2$, belong to $\mathcal{G}(t)$ (defined by (2.16)) for some $t \in I^{\prime}$, then

$$
\chi_{1}-\chi_{2}=\theta_{2}-\theta_{1} \in H^{1}(\Omega) .
$$

Since $\mathcal{G}(t) \subset B V(\Omega ;\{-1,1\})$, then (4.29) yields

$$
\chi_{1}=\chi_{2} \quad \text { or } \quad \chi_{1}=-\chi_{2} \equiv \pm 1 .
$$

Since

$$
\mathscr{G}\left(t, u_{1}\right)=\mathscr{G}\left(t, u_{2}\right)
$$

the last case implies that

$$
\int_{\Omega} w(x, t) d x=-\int_{\Omega} w(x, t) d x=0 .
$$

On the other hand, passing to the limit in (4.16) and recalling (4.22), we get $\int_{\Omega} w(x, t) d x=\rho(t) \neq 0$ for every $t \in I^{\prime}$, and so we conclude that $\mathcal{G}(t)$ is nondegenerate w.r.t. $L$ for every $t \in I^{\prime}$.

Acknowledgment. I wish to thank Pierluigi Colli for introducing me to the topic of this paper and for several discussions on this subject.

\section{REFERENCES}

[BBGT88] C. Baiocchi, G. Buttazzo, F. Gastaldi, and F. Tomarelli, General existence theorems for unilateral problems in continuum mechanics, Arch. Rational Mech. Anal. 100 (1988), no. 2, 149-189. MR 88k:73014

[CV77] C. Castaing and M. Valadier, Convex analysis and measurable multifunctions, Springer, Berlin-New York, 1977. MR 57:7169

[Dal93] G. Dal Maso, An introduction to $\Gamma$-convergence, Progress in Nonlinear Differential Equations and Their Applications, vol. 8, Birkhäuser, Boston, 1993. MR 94a:49001

[Gag61] E. Gagliardo, A unified structure in various families of function spaces. Compactness and closure theorems, Proc. Internat. Sympos. Linear Spaces (Jerusalem, 1960), Jerusalem Academic Press, Jerusalem, 1961, pp. 237-241. MR 24:A2836

[Giu84] E. Giusti, Minimal surfaces and functions of bounded variation, Birkhäuser Verlag, Basel, 1984. MR 87a:58041

[HS98a] K.-H. Hoffmann and V. N. Starovoitov, Phase transitions of liquid-liquid type with convection, Adv. Math. Sci. Appl. 8 (1998), no. 1, 185-198. MR 99d:35186

[HS98b] K.-H. Hoffmann and V. N. Starovoitov, The Stefan problem with surface tension and convection in Stokes fluid, Adv. Math. Sci. Appl. 8 (1998), no. 1, 173-183. MR 99d:35185 
[LM72] J.-L. Lions and E. Magenes, Non-homogeneous boundary value problems and applications. Vol. I-II, Springer-Verlag, New York, 1972, Die Grundlehren der mathematischen Wissenschaften, Band 182. MR 50:2670; MR 50:2671

[LP64] J.-L. Lions and J. Peetre, Sur une classe d'espaces d'interpolation, Inst. Hautes Études Sci. Publ. Math. no. 19, (1964), 5-68. MR 29:2627

[Luc90] S. Luckhaus, Solutions for the two-phase Stefan problem with the Gibbs-Thomson Law for the melting temperature, European Journal of Applied Mathematics 1 (1990), 101-111. MR 92i:80004

[Mod86] L. Modica, Gradient theory of phase transitions and minimal interface criterion, Arch. Rational Mech. Anal. 98 (1986), 123-142. MR 88f:76038

[PS93] P.I. Plotnikov and V.N. Starovoitov, The Stefan problem with surface tension as the limit of a phase field model, Differential Equations 29 (1993), 395-404. MR 94f:35155

[Rud87] W. Rudin, Real and complex analysis, third ed., McGraw-Hill Book Co., New York, 1987. MR 88k:00002

[Sch00] R. Schätzle, The quasistationary phase field equations with Neumann boundary conditions, J. Differential Equations 162 (2000), no. 2, 473-503. MR 2001b:35143

[Vis96] A. Visintin, Models of phase transitions, Progress in Nonlinear Differential Equations and Their Applications, vol. 28, Birkhäuser, Boston, 1996. MR 98a:80006

[Yos80] K. Yosida, Functional analysis, sixth ed., Springer-Verlag, Berlin, 1980, Die Grundlehren der Mathematischen Wissenschaften, Band 123. MR 82i:46002

Dipartimento di Matematica "F. Casorati", Università di Pavia. Via Ferrata, 1. I27100 Pavia, Italy

E-mail address: savare@ian.pv.cnr.it

$U R L:$ http://www.ian.pv.cnr.it/ savare 